\title{
Tropical Medicine
}

\section{Liver abscess in the tropics: experience in the University Hospital, Kuala Lumpur.}

\author{
K.L. Goh, N.W. Wong, M. Paramsothy, M. Nojeg and K. Somasundaram \\ Department of Medicine, Faculty of Medicine, University of Malaya, 59100 Kuala Lumpur, Malaysia.
}

\begin{abstract}
Summary: We reviewed 204 cases of liver abscess seen between 1970 and 1985 . Ninety were found to be amoebic, 24 pyogenic and one tuberculous. The cause of the abscesses in the remaining 89 patients was not established. The patients were predominantly male, Indians, and in the 30-60 age group. The majority of patients presented with fever and right hypochondrial pain. The most common laboratory findings were leucocytosis, hypoalbuminaemia and an elevated serum alkaline phosphatase. Amoebic abscesses were mainly solitary while pyogenic abscesses were mainly multiple. Complications were few in our patients and included rupture into the pleural and peritoneal cavities and septicaemic shock. An overall mortality of $2.9 \%$ was recorded.

The difficulty in diagnosing the abscess type is highlighted. The single most important test in helping us diagnose amoebic abscess, presumably the most common type of abscess in the tropics, is the Entamoeba histolytica antibody assay. This test should be used more frequently in the tropics.
\end{abstract}

\section{Introduction}

Liver abscess is a fairly common disease in Malaysia. Balasegaram ${ }^{1}$ reported 442 cases seen over a 15 -year period. While it can be said that his experience is that of a referral surgical centre, many cases of liver abscess are also seen and treated in smaller hospitals throughout the country.

The common types of liver abscess seen are amoebic and pyogenic abscess. Although it is often presumed that the majority of abscesses seen in the tropics are amoebic in origin, the evidence incriminating Entamoeba histolytica as the causative agent has seldom been proven. ${ }^{2}$ Similarly, cultures for pyogenic organisms are often negative.

We describe our experience with 204 cases of liver abscess seen from 1970-1985 in the University Hospital, Kuala Lumpur.

\section{Materials and methods}

A definitive diagnosis of liver abscess was made based on one or any combination of the following - surgical drainage or aspiration of pus from the liver or at

Correspondence: K.L. Goh, M.B., B.S.(Mal), M.R.C.P. (UK)

Accepted: 29 January 1987 autopsy, radionuclide scanning, ultrasonography or computed tomographic (CT) scanning.

Patients were considered to have an amoebic liver abscess when (i) $E$. histolytica trophozoites were demonstrated in a biopsy of the abscess wall; (ii) $E$. histolytica trophozoites were demonstrated in the pus; (iii) E. histolytica antibody titres were greater than 1:64; (iv) anchovy sauce was drained from the abscess; (v) E. histolytica trophozoites or cysts were isolated in the stools with or without a history of dysentery; and (vi) patients responded to treatment with anti-amoebic drugs alone.

Patients were considered to have a pyogenic abscess when pus and/or blood cultures grew pyogenic organisms, provided the above criteria for amoebic abscess were negative and there was no other obvious source of infection.

One patient was diagnosed to have a tuberculous abscess when biopsy of the abscess wall demonstrated granulomas with acid-fast bacilli. There was no evidence of pulmonary or miliary tuberculosis.

Patients were placed in an indeterminate group when the criteria for diagnosis of amoebic or pyogenic abscess were not present or known. 
Table I Clinical presentation

\begin{tabular}{|c|c|c|c|c|}
\hline & $\begin{array}{c}\text { Amoebic } \\
n=90\end{array}$ & $\begin{array}{c}\text { Pyogenic } \\
n=24\end{array}$ & $\begin{array}{c}\text { Indeterminate } \\
n=89\end{array}$ & $\begin{array}{c}\text { All abscesses } \\
n=204\end{array}$ \\
\hline Fever with chills and rigors & 80.0 & 79.2 & 88.8 & 82.7 \\
\hline Right hypochondrial pain & 77.7 & 50.0 & 78.7 & 68.8 \\
\hline Epigastric pain & 14.4 & 33.3 & 14.6 & 20.8 \\
\hline Diarrhoea or dysentery & 19.9 & 8.3 & 15.9 & 14.7 \\
\hline Jaundice & 24.4 & 16.7 & 17.0 & 19.4 \\
\hline Tender hepatomegaly & 90.0 & 87.5 & 90.9 & 89.8 \\
\hline $\begin{array}{l}\text { Right basal effusion or crackles/ } \\
\text { rub }\end{array}$ & 39.9 & 8.3 & 32.9 & 27.0 \\
\hline
\end{tabular}

${ }^{*}$ Figures are in percentages of the abscess type.

\section{Results}

Of 204 patients reviewed, $90(44.1 \%)$ were found to have amoebic abscess, $24(11.8 \%)$ pyogenic abscess, one $(0.5 \%)$ tuberculous abscess while $89(43.6 \%)$ were considered indeterminate.

The majority of patients were male, with a male to female ratio of $6: 1$. Indians were the predominant racial group affected and comprised $49.5 \%$ of all patients compared with $23.2 \%$ of all hospital admissions $(P<0.01)$. The age of patients ranged from 2 years to 84 years. The peak incidence occurred in the $30-60$ years age-group.

The clinical presentation of liver abscess is as shown (Table I). The common presenting clinical features for all abscess types were fever with chills and rigors, right hypochondrial pain and a tender hepatomegaly. Only a small proportion $(20 \%)$ of patients had jaundice. Right lower chest signs, chiefly a right basal effusion were seen in $40 \%$ of the patients with amoebic abscess and in $32.9 \%$ of patients in the indeterminate group. The incidence is, however, much lower in the pyogenic group $(8.3 \%)$.

The haemoglobin level was less than $11 \mathrm{~g} / 100 \mathrm{ml}$ in $53.5 \%$ of the patients and the total white cell count was elevated to above $11 \times 10^{9} / 1$ in $81 \%$. A low serum albumin level of less than $35 \mathrm{~g} / \mathrm{l}$ and an elevated serum alkaline phosphatase of greater than $150 \mathrm{IU} / 1$ were found in about $80 \%$ of patients. Serum bilirubin and alanine transaminase levels were raised in $29 \%$ and $24 \%$ respectively. There was no significant difference between the patient groups.

The most frequently used radiological modality in diagnosis and localization of abscess was radionuclide scanning, utilized in three-quarters of the patients. Recently, ultrasonography has been increasingly utilized.

Table II shows the location of abscesses in the various subgroups. Of the amoebic abscesses $86.5 \%$ were single, with the majority in the right lobe $(77.7 \%$ of the total). On the other hand $62.6 \%$ of pyogenic abscesses were multiple. The figures for the indeterminate group approximated that of amoebic abscesses.

The bacteriology of pyogenic abscess is as shown in Table III. Escherichia coli and Klebsiella species were the most common pyogenic oganisms and together accounted for more than half of the organisms isolated.

Predisposing factors were looked for in the 24 patients with pyogenic abscess. In 6 , there was evidence of biliary disease while there were no obvious

Table II Abscess location*

\begin{tabular}{lccc}
\hline & Amoebic & Pyogenic & Indeterminate \\
\hline Single abscess & & & \\
Right lobe & 77.7 & 25.0 & 64.0 \\
Left lobe & 8.8 & 12.5 & 13.5 \\
Multiple abscesses & & & \\
Right lobe & 8.8 & 4.2 & 7.9 \\
Left lobe & 1.1 & 4.2 & 3.4 \\
Both lobes & 3.3 & 54.2 & 11.2 \\
\hline
\end{tabular}

*All figures in percentages of the particular abscess type. 
Table III Bacteriology of pyogenic abscess

\begin{tabular}{lcc}
\hline & No. & $(\%)$ \\
\hline Escherichia coli & 8 & 33.3 \\
Klebsiella sp. & 6 & 25.0 \\
Enterobacter sp. & 3 & 12.5 \\
Streptococcus milleri & 2 & 8.3 \\
Serratia marcesens & 2 & 8.3 \\
Streptococcus faecalis, Bac- & 1 each & \\
teroides sp. Citrobacter sp. & & \\
Pseudomonas pseudomallei & & \\
\hline
\end{tabular}

precipitating factors in the remainder. In the indeterminate group, abscesses followed a perforated appendix, a septic abortion and in 2 patients, biliary disease.

No standard treatment protocol was adopted in our patients. The majority were treated with both antibiotics and anti-amoebic drugs as soon as the diagnosis of liver abscess was made. The patients were usually referred early for a surgical opinion. The indications for needle aspiration or surgical drainage included non-resolution of clinical signs and symptoms with adequate medical treatment, signs of rupture or impending rupture of the abscess and a large size of abscess exceeding $10 \mathrm{~cm}$, especially when located in the left lobe.

In the amoebic abscess group, $44.4 \%$ of patients had open drainage and $17.7 \%$ had needle aspiration of their abscesses carried out. In the pyogenic group, $70.8 \%$ of abscesses were surgically drained and $8.3 \%$ needle aspirated. However, in the large group with indeterminate abscess $27.0 \%$ of patients had open drainage and $13.5 \%$, needle aspiration performed.

Complications were uncommon in our group of patients; 7 patients had perforations into the peritoneal cavity, 4 had perforations into the pleural cavity. Two patients with amoebic abscess developed severe fulminating colitis and septicaemic shock and succumbed to the illness; both patients were aborigines. Three patients in the pyogenic group and one in the indeterminate group died following septicaemic shock. The overall mortality rate was $2.9 \%$.

\section{Discussion}

While the diagnosis of liver abscess is reliably made on clinical grounds and easily confirmed by radionuclide scanning, ultrasonography or CT scanning, the diagnosis of abscess type is difficult. This is reflected by the large number of patients placed in the indeterminate group. There are several reasons for this problem. Not all abscesses are drained or aspirated yielding pus for examination. Even if pus were available, amoeba can only be located with difficulty. Biopsy of the abscess wall is not always done at open drainage, and again, if done, amoeba may not be found because they disintegrate and disappear quickly with preceding antiamoebic treatment. ${ }^{3}$ Pus cultures for pyogenic organisms are affected by previous antibiotic therapy - often the case with our patients who have sought treatment before coming to hospital. Furthermore, pus cultures for anaerobic organisms are not routinely done. This is pertinent as there are reports that anaerobes may account for up to $38 \%$ of all pyogenic abscesses. ${ }^{4}$ Associated or preceding dysentery in patients with amoebic liver abscess is uncommon with our patients and so does not help in making a diagnosis of amoebic abscess. Anchovy sauce when aspirated or drained is highly characteristic of amoebic abscess. However, it may not be found in all amoebic abscesses.

Entamoeba histolytica antibody assay is useful in confirming the diagnosis of amoebic abscess as tissue invasion by the amoeba induces a strong antibody response. ${ }^{5}$ The indirect immunofluorescent method is used in our laboratory and has been found to be highly sensitive, with a reactivity of $96 \%$ among confirmed cases of liver abscess. ${ }^{6}$ Unfortunately this test is still not readily available locally. Only 4 sera were tested in the indeterminate group; 2 were non-reactive while 2 had titres of 1 in 16 which were considered not significant. It is likely therefore that many cases of amoebic abscess were left undiagnosed in the indeterminate group.

The sex and race distribution of our group of patients was very similar to other reports from this region. ${ }^{8,9}$ Male preponderance is noted in our series. Indians were the predominant racial group affected overall. This is not surprising as the majority of Indian patients seeking treatment in the hospital come from outlying areas around Kuala Lumpur, where hygiene is poor.

Clinical signs and symptoms were fairly reliable and consistent in helping us diagnose liver abscess. However, the clinical features of amoebic and pyogenic abscesses were on the whole remarkably similar and did not help us distinguish between the two. An exception is the occurrence of right basal lung signs in $40 \%$ of patients with amoebic abscess which was only present in $8.3 \%$ of patients with pyogenic abscess. This can perhaps be explained by the fact that the majority of amoebic abscesses were in the right lobe of the liver where it would be most likely to cause right basal lung signs especially when located in the diaphragmatic surface. The higher proportion of abscesses in the right lobe also explains the higher ratio of right hypochondrial pain to epigastric pain in amoebic abscess. On the other hand, in pyogenic abscess, where a significant proportion of abscesses were in the left lobe or in both lobes, epigastric pain was present in an increased proportion of patients.

We have observed that the number and location of 
abscesses were useful in helping to distinguish between amoebic and pyogenic abscess. A single right lobe abscess was more likely to be amoebic while multiple abscesses in both lobes were more likely to be pyogenic. These findings are consistent with the observations of others., 9

Not unexpectedly, the Gram-negative bacilli, E. coli and Klebsiella were the most common pyogenic organisms isolated. Bacteroides was found in only one patient but as noted before, the detection of anaerobic organisms is hampered by collection and inadequate anaerobic culturing technique. One patient had systemic melioidosis with development of multiple liver abscesses; Pseudomonas pseudomallei was cultured from both blood and pus.

While associated predisposing factors were found in only six patients with pyogenic abscess, we found four patients with definite evidence of biliary disease or intra-abdominal sepsis in the indeterminate group. These patients are likely to have had pyogenic abscesses.

The overall morbidity and mortality rates in our patients were low which could perhaps be attributed to a prompt and aggressive approach to treatment. Perforations into the peritoneal and pleural cavities

\section{References}

1. Balasegaram, M. Management of hepatic abscess. Curr Probl Surg 1981, 18: 282-340.

2. Vijendran, $M$. The diagnosis and current treatment of liver abscess. Med J Malaysia 1977, 32: 133-138.

3. Manson-Bahr, P.E.C. \& Apted, F.I.C. Amoebiasis, giardiasis and balantidiasis. In Manson's Tropical Diseases. Bailliere Tindall, Edinburgh, London, 1982, pp. 121 145.

4. Perera, M.R. Presentation, diagnosis and management of liver abscess. Lancet 1980, ii: 629-632.

5. Krupp, M.I. Antibody response in intestinal and extraintestinal amoebiasis. Am J Trop Med Hyg 1970, 19: 57 62. but not into the pericardial sac were documented. It is interesting to report two cases of fulminating amoebic colitis, an uncommon complication of amoebiasis, occurring with liver abscess. Deaths among our patients usually followed overwhelming septicaemia.

Finally, we have also noted the remarkable similarity in the epidemiological data, clinical features, and laboratory and radiological results of the amoebic and indeterminate groups. It would be tempting to presume that the majority of the patients in the indeterminate group were amoebic. As amoebic serological tests have been shown to be highly sensitive, a more conclusive diagnosis of amoebic liver abscess could have been made if these tests had been performed on more patients. This would have been extremely helpful in planning more precise and definitive treatment of the disease, thereby cutting down on costs and undesirable side effects of therapy.

\section{Acknowledgements}

We would like to thank Mr Low Ting, Department of Medicine, University of Malaya, for helping us prepare the manuscript.
6. Thomas, V., Sinniah, B. \& Yap, P.L. Assessment of the sensitivity, specificity, and reproducibility of the indirect immunofluorescent technique for the diagnosis of amebiasis. Am J Trop Med Hyg 1980, 30 (1): 57-62.

7. Fock, K.M., Ong, N.T. \& Chua, K.L. Liver abscess: a two-year study. Singapore Med J 1985, 26: 350-353.

8. Teh, L.B., Ng, H.S., Kwok, K.C. et al. Liver abscess - a clinical study. Ann Acad Med Singapore 1986, 15 (2): $176-000$.

9. May, R.P. Difficulties in differentiating amoebic from pyogenic liver abscess. Arch Int Med 1967, 119: 69-74. 